\title{
HOLONOMY GROUPS OF HYPERSURFACES
}

\author{
SHOSHICHI KOBAYASHI
}

The restricted homogeneous holonomy group of an $n$-dimensional Riemannian manifold is a connected closed subgroup of the proper orthogonal group $S O(n)$ [1]. In this note we shall prove that the restricted homogeneous holonomy group of an $n$-dimensional compact hypersurface in the Euclidean space is actually the proper orthogonal group $S O(n)$ itself. This gives a necessary (of course, not sufficient) condition for the imbedding of an $n$-dimensional compact Riemannian manifold into the $(n+1)$-dimensional Euclidean space.

The method used here shows how the theory of connections in fibre bundles is efficacious for problems in classical differential geometry. In fact, the essential part in this paper is the notion of indused cnnnection, which is outside of the frame of classical differential geometry.

I would like to say thanks to Professor Allendoerfer and Dr. Forrester for their valuable suggestions.

\section{Holonomy groups}

Let $P$ be a principal fibre bundle over a manifold $M$ with Lie structure group $G$ and with projection $\pi$. Let $\omega$ be a $g$-valued linear differential form on $P$ defining an infinitesimal connection in $P[3]$, where $g$ is the Lie algebra of $G$. Let $u_{0}$ be a point in $P$. If $c$ is a closed curve in $M$ starting from $x_{0}=\pi\left(u_{0}\right)$, then the point $c\left(u_{0}\right)$ obtained by the parallel displacement of $u_{0}$ along the curve $c$ is in the same fibre as $u_{0}$; hence there is a unique element $s$ in $G$ which maps $u_{0}$ to $c\left(u_{0}\right)$ :

$$
c\left(u_{0}\right)=u_{0} s .
$$

(The structure group $G$ acts on $P$ on the right, as $P$ is a principal fibre bundle.) The set of all elements $s$ obtained in this way forms a subgroup (not necessarily closed) of $G$, which is called the holonomy group of the connection $\omega$ with refer-

Received May 20, 1955. 
ence point $u_{0}$. We shall denote it by $h\left(M, u_{0}\right)$. If we consider only the closed curves which are homotopic to zero, then we obtain a normal subgroup $h^{0}\left(M, u_{0}\right)$ of $h\left(M, u_{0}\right)$, which is called the restricted holonomy group. It is just the connected component of the unit of $h\left(M, u_{0}\right)$,

Let $U$ be a connected open set containing $x_{0}$. If we consider only the closed curves contained in $U$, we obtain a subgroup of $h\left(M, u_{0}\right)$, which will be denoted by $h\left(U, u_{0}\right)$. Take a decreasing sequence of connected open sets in $M$

$$
U_{1} \supset U_{2} \supset \ldots \text { such that } \cap U_{i}=\left\{x_{0}\right\} \text { and } U_{i} \supset \bar{U}_{i+1} .
$$

Consider the group

$$
h^{*}\left(u_{0}\right)=\cap h^{0}\left(U_{i}, u_{0}\right)
$$

where $h^{0}\left(U_{i}, u_{0}\right)$ is the connected component of $h\left(U_{i}, u_{0}\right)$. This Lie group $h^{*}\left(u_{0}\right)$ is called the local holonomy group at $u_{0}$ [4]. It is known that, if the dimension of $h^{*}\left(u_{0}\right)$ is constant over $P$, then $h^{*}\left(\boldsymbol{u}_{0}\right)$ coincides with the restricted holonomy group $h^{0}\left(M, u_{0}\right)$ [4].

\section{Induced connection}

Let $Q$ be a principal fibre bundle over a manifold $S$ with group $G$ and with projection $\pi^{\prime}$. If $\omega^{\prime}$ is a $g$-valued linear differential form on $Q$ defining a connection in $Q$ and if $\tilde{F}$ is a bundle map of $P$ into $Q$, then the induced form $\omega$ $=\widetilde{f}^{*}\left(\omega^{\prime}\right)$ defines a connection in $P$. Let $h\left(S, v_{0}\right)$ be the holonomy group of the connection defined by $\omega^{\prime}$, where $v_{0}=\widetilde{f}\left(u_{0}\right)$. We shall show that $h\left(M, u_{0}\right)$ is a subgroup of $h\left(S, v_{0}\right)$. Take an arbitrary closed curve $c$ in $M$ starting from $x_{0}$ and consider the corresponding curve $f(c)$ in $S$, where $f$ is a mapping of $M$ into $S$ induced by $\tilde{f}$. Let $\tilde{c}$ be the horizontal curve [1] in $P$ starting from $u_{0}$ and covering $c$. Then the end point of $\tilde{c}$ is the point obtained by the parallel displacement of $u_{0}$ along $c$. It is clear that the image $\tilde{f}(\tilde{c})$ of the horizontal curve $\tilde{c}$ is horizontal. Therefore the element $s$ of $G$ corresponding to the curve $c$ is the element of $G$ corresponding to the curve $f(c)$.

Thus we have proved the following

Lemma 1. The holonomy group $h\left(M, u_{0}\right)$ of the induced connection is a subgroup of the holonomy group $h\left(S, v_{0}\right)$ of the initial connection.

COROLlaRY. The restricted holonomy group $h_{0}\left(M, u_{0}\right)$ is a subgroup of $h^{0}\left(S, v_{0}\right)$. 
Now we shall prove the following

Lemma 2. Suppose that the following two conditions are satisfied:

(1) There is a connected open set $U$ in $M$ such that $f$ is a differentiable homeomorphism of $U$ into $S$;

(2) The dimension of local holonomy groups $h^{*}(v)$, where $v \in Q$, is constant on $Q$.

Then the restricted holonony group $h_{1}\left(M, u_{0}\right)$ of the induced connertion coincides with the restricted holonomy group $h_{0}\left(S, v_{n}\right)$ of the initial connection.

Proof. Since the holonomy groups with different reference points are conjugate to each other in $G$, we may assume that $\pi\left(u_{0}\right)$ is contained in $U$. In general we have the following relations:

$$
\begin{aligned}
& h^{0}\left(M, u_{0}\right) \supset h^{0}\left(U, u_{0}\right) \supset h^{*}\left(u_{0}\right) \\
& h^{0}\left(S, v_{0}\right) \supset h^{0}\left(V, v_{0}\right) \supset h^{*}\left(v_{0}\right),
\end{aligned}
$$

where $V=f(U)$.

From assumption (2) it follows [4] that $h^{0}\left(S, v_{0}\right)=h^{*}\left(v_{0}\right)$; hence

$$
h^{0}\left(S, v_{0}\right)=h^{0}\left(V, v_{0}\right)=h^{*}\left(v_{0}\right)
$$

By the same argument for Lemma 1, we obtain the following relation:

$$
h^{0}\left(U, u_{0}\right) \subset h^{0}\left(V, v_{0}\right) .
$$

As $f$ is a homeomorphism of $U$ onto $V$, we have also

$$
h^{0}\left(U, u_{0}\right) \supset h^{0}\left(V, v_{0}\right) .
$$

Therefore we have

$$
h^{0}\left(S, v_{0}\right)=h^{0}\left(V, v_{0}\right)=h^{0}\left(U, u_{0}\right) .
$$

Hence $h^{0}\left(S, v_{0}\right)$ is contained in $h^{0}\left(M, u_{0}\right)$. It follows from the corollary to Lemma 1 that $h^{0}\left(S, v_{0}\right)=h^{0}\left(M, u_{0}\right)$.

\section{Spherical indicatrix}

Let $M$ be an orientable hypersurface in the $(n+1)$-dimensional Euclidean space $R^{n+1}$ and $S$ the $n$-dimensional unit sphere in $R^{n+1}$ with center $O$, origin of $R^{n+1}$. $M$ and $S$ have the Riemannian metrics induced from that of $R^{n+1}$.

We shall use the following notations: 
$T_{x}(M)$ (resp. $\left.T_{y}(S)\right)$ : the tangent space to $M$ (resp. $S$ ) at apoint $x$ in $M$ (resp. $y$ in $S$ ).

$G_{x}$ (resp. $H_{y}$ ): the set of all orthonormal frames on $M$ at $x$ (resp. on $S$ at $y)$. Both $G_{x}$ and $H_{y}$ are isomorphic to $O(n)$.

$P($ resp. $Q)$ : the set of all orthonormal frames on $M$ (resp. $S$ ).

$$
P=\bigcup_{x \in M} G_{x}, \quad Q=\bigcup_{y \in S} H_{y} .
$$

$\pi$ (resp. $\left.\pi^{\prime}\right)$ : the projection of $P$ onto $M$ (resp. of $Q$ onto $S$ ) such that

$$
\pi\left(G_{x}\right)=x, \quad \pi^{\prime}\left(H_{y}\right)=y .
$$

It is well known that $P$ (resp. $Q$ ) is a principal fibre bundle over $M$ (resp. $S$ ) with group $O(n)$ and with projection $\pi$ (resp. $\pi^{\prime}$ ).

Let $\xi_{x}$ be a unit normal vector to $M$ at $x$ chosen in such a way that $\xi_{x}$ becomes a continuous vector field (this is possible, since $M$ is orientable). Let $f(x)$ be the end point of the unit vector in $R^{n+1}$ with origin $O$, which is parallel to the vector $\xi_{x}$. Then $f(x)$ is a point on $S$. Thus we obtain a mapping $f$ of $M$ into $S$. Let $u$ be an element of $G_{x}$, i.e., an orthonormal frame on $M$ at $x$. By the parallel displacement in the Euclidean space $R^{n+1}$, we obtain from $u$ an orthonormal frame $v$ on $S$ at $f(x)$. We denote by $\tilde{f}$ the mapping $u \rightarrow v$ of $P$ into $Q$. Clearly $\tilde{f}$ is a bundle map.

The Riemannian metric on $S$ defines a unique linear (so-called affine) connection without torsion on $S$. Let $\omega^{\prime}$ be the $g$-valued linear differential form on $Q$ defining this linear connection, where $g$ is the Lie algebra of the orthogonal group $O(n)$. The connection defined by the induced form $f^{*}\left(\omega^{\prime}\right)$ coincides with the connection defined by the Riemannian metric on $M$. This fact can be verified by straightforward calculation [5]; however it follows also from the following geometrical consideration. Let $\bar{x}$ be an arbitrary tangent vector to $M$ at $x$ and $c$ any curve in $M$ starting from $x$. Let $y$ be the tangent vector to $S$ at $f(x)$ obtained by the parallel displacement of $\bar{x}$ in the Eucliden space $R^{n+1}$. Let $\bar{y}^{\prime}$ be the tangent vector to $S$ obtained by the parallel displacement of $\bar{y}$ along $f(c)$ with respect to the Riemannian connection on $S$. Let $\bar{x}^{\prime}$ be the tangent vector to $M$ at the end point of the curve $c$ obtained by the parallel displacement of $\bar{y}^{\prime}$ in the Euclidean space $R^{n+1}$. Then $\bar{x}^{\prime}$ is the tangent vector to $M$ obtained by the parallel displacement of $\bar{x}$ along $c$ with: respect to the Riemannian connection on $M$. This is the geometrical meaning of the induced connection. And 
this is also the classical interpretation of parallel displacement on the Riemannian manifold imbedded in $R^{n+1}$. Therefore the induced connection coincides with the connection defined by the Riemannian metric on $M$.

\section{Holonomy group of spheres}

The orthogonal group $O(n+1)$ acts transitively on $S$ as the group of isometries. Therefore $S$ has the homogeneous structure and assumption (2) of Lemma 2 is satisfied.

Since $S$ is an irreducible symmetric Riemannian space in the sense of $\mathrm{E}$. Cartan, the restricted holonomy group is equal to the connected component of the isotropic subgroup of the group of isometries. As the isotropic subgroup of $O(n+1)$ acting on $S$ is $O(n)$, the restricted holonomy group of $S$ is the proper orthogonal group $S O(n)$.

\section{Dénouement}

In the preceding section we showed that assumption (2) of Lemma 2 is satisfied; in this section we shall prove assumption (1).

Let $\rho(x)$ be the Euclidean distance between the origin $O$ and $x \in M$. As $M$ is compact, the function $\rho$ attains its maximum at a certain point $x_{0}$ of $M$. Then $M$ is inside of the sphere with center $O$ and with radius $\rho\left(x_{0}\right)$ and is tangent to the sphere at $x_{0}$. Therefore the mapping $f: M \rightarrow S$ is a homeomorphism in a neighborhood of $x_{0}$ (note that the second fundamental form is definite at $x_{0}$ ).

Now it follows from Lemma 2 that the restricted holonomy group of $M$ is $S O(n)$. The condition of orientability of $M$ was used only for the global definition of $f$. We saw however that the mapping $f$ defined in a neighborhood of $x_{0}$ is sufficient for our purpose.

According as $M$ is orientable or not, the homogeneous holonomy group of $M$ is connected or not. Hence we have proved the following

Theorem. Let $M$ be a compact $n$-dimensional Riemannian manifold imbedded isometrically in the $(n+1)$-dimensional Euclidean space. Then the homogeneous holonomy group of $M$ is the proper orthogonal group $S O(n)$ or the orthogonal group $O(n)$, according as $M$ is orientable or not. 


\section{BIBLIOGRAPHY}

[1] Ambrose, W-Singer, I. M., A theorem on holonomy, Trans. Amer. Math. Soc. 75 (1953), p. $428-443$.

[2] Borel, A.-Lichnerowicz, A., Groupes d'holonomie des variétés riemanniennes, C. $R$. Acad. Sci. Paris 234 (1952), p. 1835-1837.

[3] Ehresmann, C., Les connexions infinitésimales dans un espace fibré différentiable, Colloque de topologie, Bruxelles (1950).

[4] Nijenhuis, A., On the holonomy groups of linear connections, Nederl. Akad. Wetensch. Proc. A 56 (1953), p. 233-249.

[5] Kobayashi, S., Induced connections and imbedded Riemannian spaces, this journal.

University of Washington 